\title{
Integration of fiber-tip cavities in single-ion traps for quantum sensing
}

\author{
Peter Horak*a ${ }^{*}$, Nina Podoliak ${ }^{\mathrm{a}}$, Hiroki Takahashi ${ }^{\mathrm{b}}$, Matthias Keller ${ }^{\mathrm{b}}$ \\ ${ }^{a}$ Optoelectronics Research Centre, University of Southampton, Southampton SO17 1BJ, United \\ Kingdom; \\ ${ }^{\mathrm{b}}$ Department of Physics and Astronomy, University of Sussex, Brighton BN1 9QH, United \\ Kingdom; \\ *peh@orc.soton.ac.uk
}

\begin{abstract}
We investigate geometries for efficient coupling of single ions to fiber-coupled light fields for applications in quantum sensing, quantum metrology, and quantum information processing. Specifically, we discuss the integration of fiber-tip microcavities into radio-frequency ion traps. The distortions of the trapping fields induced by the presence of the optical fibers are simulated for a range of ion trap geometries and the most promising arrangements are identified. Finally, we investigate the use of fiber-tip microcavities with non-spherical mirrors for enhanced ion-light coupling at the center of the trap by appropriate shaping of the cavity modes.
\end{abstract}

Keywords: Optical microcavities, fiber cavities, quantum sensing, quantum information processing, ion traps.

\section{INTRODUCTION}

Quantum-enhanced sensing, where the quantum mechanical properties of light and matter are exploited to create sensors of unprecedented sensitivity, is currently considered the most advanced area in the wider field of novel quantum technologies. Of particular interest are sensors based on ultra-cold ions ${ }^{1,2}$ trapped in radio-frequency (rf) ion traps because of their robustness, potential for integration on silicon chips, and unparalleled long coherence times. Similar iontrap systems are also prime contenders for future atomic clock standards ${ }^{3}$, for scalable quantum information processing and computing ${ }^{4}$, and for quantum simulators ${ }^{5}$.

For most of these applications the trapped ions also need to interact with light fields for optical cooling of the ion motion and for the initial quantum state preparation. Finally, optical readout of the electronic state of the ion is required to extract the result of the sensor or quantum data operation from the quantum system and make it accessible macroscopically. To date, much of this optical manipulation of trapped ions is achieved by free-space optics. However, for future chip-based integration, for scalability, and for improved efficiency it is highly desirable to replace these optical links by fiber optics.

(a)
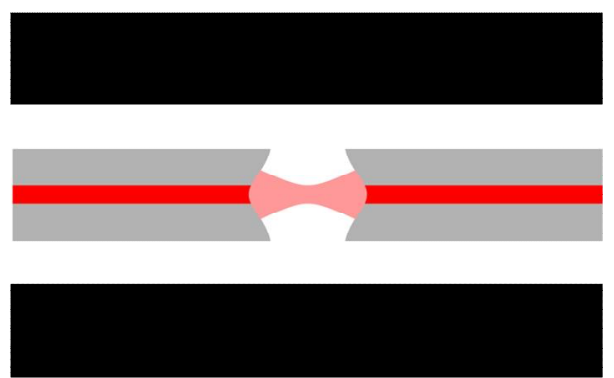

(b)
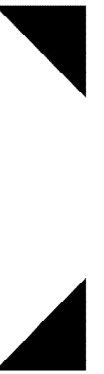
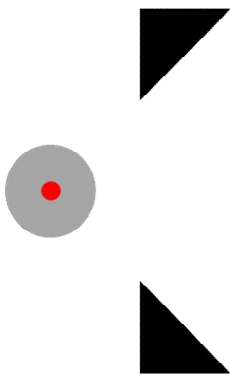

Figure 1. (a) Longitudinal and (b) cross section schematic of a blade-type ion trap with integrated fiber-tip optical cavity. Black: metal electrodes generating the ion trapping potential; grey: optical fiber; red: optical fiber mode and cavity mode. Dimensions not to scale.

In our work, we investigate the integration of optical fiber microcavities into rf ion traps. In particular, we focus on fibertip cavities where high reflectivity mirrors are machined onto the ends of two optical fibers forming a small-scale (sub$\mathrm{mm}$ ) optical resonator ${ }^{6}$ which is then inserted into the $\mathrm{rf} \mathrm{trap}^{7}$. A schematic of the setup is shown in Fig. 1. By numerical 
simulations we address two questions: (i) What is the optimum geometry for such a fiber-cavity coupled ion trap to minimize the distortion of the $\mathrm{rf}$ trapping field by the presence of the dielectric fiber material ${ }^{8}$, and (ii) is it possible to shape the cavity mode fields to maximize the coupling of a trapped ion to the light field inside the cavity ${ }^{9}$ ?

\section{DISTORTIONS OF ION TRAPPING FIELDS BY DIELECTRIC CAVITIES}

A number of different ion traps have been proposed for quantum sensing and quantum information purposes, in particular the linear blade trap ${ }^{10}$, cylindrical endcap ${ }^{7}$ and stylus traps ${ }^{1}$, and planar surface traps ${ }^{11}$ as shown in Fig. 2 . For each of these traps we performed detailed numerical simulations using a finite element method (Comsol Multiphysics ${ }^{\circledR}$ ) to evaluate the trapping potential depth and secular frequency as a function of fiber cavity alignment (along the $\mathrm{x}$, $\mathrm{y}$, and $\mathrm{z}$ axes) and of the length of the gap between the two fiber tips forming the optical cavity ${ }^{8}$.

(a)
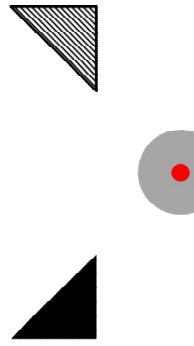
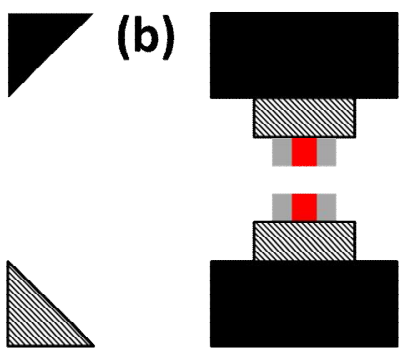

(c)
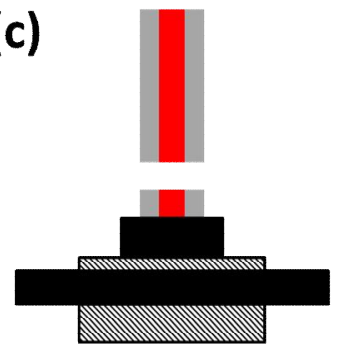

(d)
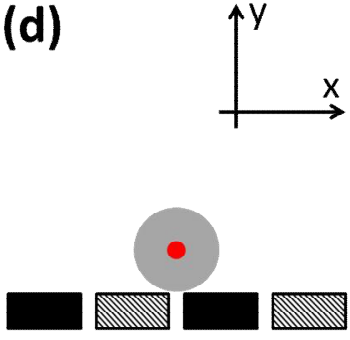

Figure 2. Ion trap geometries under investigation: (a) blade, (b) endcap, (c) stylus, (d) surface trap. Black: grounded electrodes; shaded: rf electrodes; grey: optical fibers; red: optical fiber modes. Dimensions not to scale.
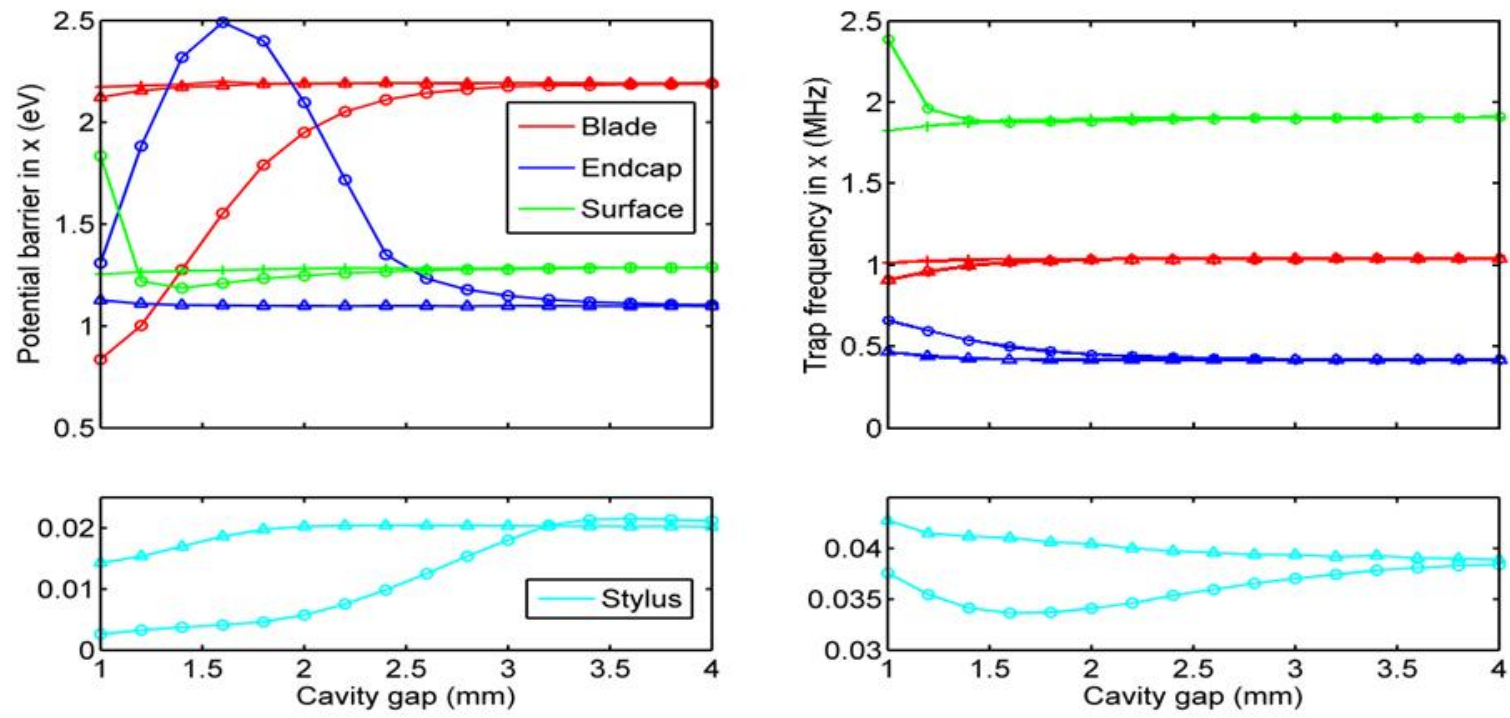

Figure 3. Simulated potential barrier (left column) and trap frequency (right column) for the four different ion trap geometries of Fig. 2 when the fiber-tip cavity is aligned along the x- (circles), y- (triangles), and z-axis (crosses).

In order to achieve a fair comparison we scale all traps to have typical gaps between the electrodes of $1 \mathrm{~mm}$. The optical fibers are modelled as cylindrical silica glass rods of $0.7 \mathrm{~mm}$ diameter. The applied rf voltage is $200 \mathrm{~V}$ and the rf frequency is $12.7 \mathrm{MHz}$. Fig. 3 shows a few selected simulation results. For cavity gaps larger than 4 mm, no distortion of the trapping fields is observed. For gap sizes decreasing towards $1 \mathrm{~mm}$ we note some significant changes in the ion trap depths. In particular, for the blade trap with the fiber-tip cavity aligned along $\mathrm{x}$, the potential depth in the $\mathrm{x}$-direction decreases from $2.2 \mathrm{eV}$ to $0.8 \mathrm{eV}$, i.e. by over $60 \%$. The stylus trap, which already exhibits very shallow trapping potentials without an integrated cavity, suffers significant further deterioration when optical fibers are introduces. On the other hand we note that trapping potentials are only slightly perturbed when the optical fibers are aligned along minima of the trapping fields (e.g. along the z-axis in the blade and surface traps) or are shielded inside the metal electrodes in the cylindrical geometries (fibers along the $y$-axis in the endcap trap). The trap frequencies, right column in Fig. 3, are 
generally less affected by the integration of fiber cavities into the traps than the potential depths, but again the effects are minimized in the same geometries and alignments as for the potential depths. Similar results were also obtained for the trap depths and frequencies in the y direction.

\section{OPTIMIZED CAVITY COUPLING BY NON-SPERICAL MIRRORS}

The fiber-tip microcavities are fabricated by laser machining with a $\mathrm{CO}_{2}$ laser ${ }^{6}$. The laser is used to ablate fiber material at the end of an optical fiber, thus creating a spatial ablation profile that follows the Gaussian beam profile of the laser. The fiber tips are then coated with a high reflectivity dielectric Bragg mirror, allowing for cavity finesses of the order of $10^{5}$. The cavity mirrors therefore deviate from the spherical shape that is conventionally used for microcavities. To investigate the effect such a Gaussian mirror profile has on the coupling of trapped ions to cavity photons, we calculate the cavity eigenmodes using a semi-analytical theory ${ }^{9,12}$.
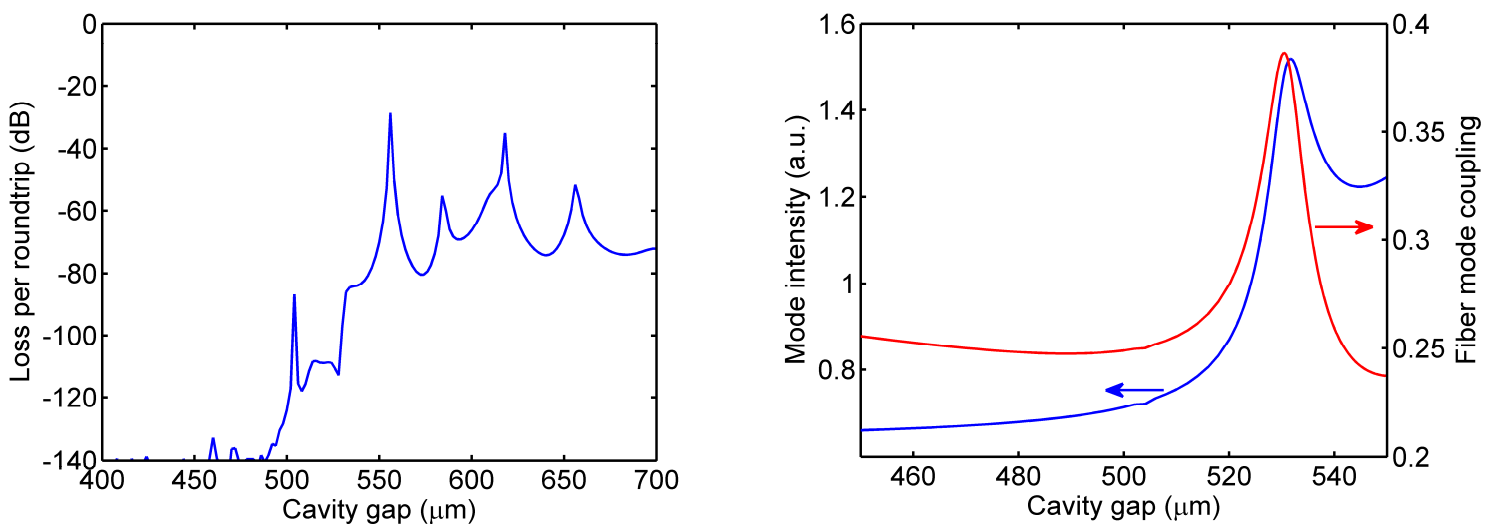

Figure 4. Simulated lowest order eigenmode of a fiber-tip cavity with Gaussian shaped mirrors of $500 \mu \mathrm{m}$ radius of curvature and indentation depth of $5 \mu \mathrm{m}$ versus cavity length. (Left) Loss per roundtrip. (Right) Mode intensity at the cavity center (blue, left axis) and coupling efficiency into single-mode optical fiber (red, right axis).

Fig. 4 shows the results of such simulations for a specific Gaussian mirror profile with $5 \mu \mathrm{m}$ indentation depth, a central radius of curvature of $\mathrm{R}=500 \mu \mathrm{m}$, and a wavelength of $866 \mathrm{~nm}$ used for $\mathrm{Ca}^{+}$ion trap experiments. For cavity lengths $\mathrm{L}$ (=gap size between the two fibers forming the cavity) smaller than the radius of curvature, the Gaussian-shaped cavity is equivalent to a spherical mirror cavity since the mode expansion on the mirrors is sufficiently small such that the deviation from the spherical shape is insignificant. In the confocal limit at $\mathrm{L} \approx \mathrm{R}$ the cavity is marginally stable and the roundtrip losses of the fundamental mode show a peak. For larger cavity lengths, we then note a series of higher-loss resonances. At these positions the non-spherical shape of the mirrors leads to resonant coupling of the lowest LaguerreGaussian (LG) mode to discrete higher order LG modes.

The first of these resonances occurs around $\mathrm{L} \approx 530 \mu \mathrm{m}$ and is formed by a resonant superposition of the fundamental $\mathrm{LG}$ mode and the second higher order LG mode. The round-trip losses at this point increase sharply to around $-80 \mathrm{~dB}$. However, this still allows for the formation of high-finesse cavities. Simultaneously we note that at these parameters the intensity of the cavity mode at the center of the cavity is increased by more than a factor of two. A trapped ion positioned there will thus be coupled to the cavity field over twice as strongly as it would be in an equivalent spherical-mirror microcavity.

Finally, we look at the coupling of the cavity eigenmode into the optical fibers forming the cavity. A large mode overlap between cavity mode and fiber mode is desirable to minimize the losses when interrogating the trapped ion through the optical fiber on a single quantum level. Assuming that the cavity is formed of standard single-mode optical fiber with waist $3 \mu \mathrm{m}$, the cavity mode of Fig. 4 is generally much larger than the fiber mode, leading to a low mode overlap factor of $\sim 0.25$. Interestingly however, the superposition mode formed at $\mathrm{L} \approx 530 \mu \mathrm{m}$ in a Gaussian-mirror cavity exhibits larger mode coupling of close to 0.4 . This mode therefore has stronger coupling to the ion as well as better output coupling into the connecting optical fibers. 


\section{CONCLUSIONS}

We studied the integration of optical fiber microcavities into radio-frequency ion traps for applications in quantum sensing, quantum metrology, and quantum information. First, we investigated how the rf trapping fields are distorted when dielectric materials, such as optical fibers, are inserted into various trapping geometries. We identified two ways to minimize these distortions: either by aligning the optical fibers along axes of minimum electrical field, or by shielding the fibers inside metal electrodes. Second, we analyzed the optical modes of fiber-tip cavities with non-spherical mirrors, in particular Gaussian-shaped mirrors as they occur naturally by laser machining. We found that the cavity mode fields can be enhanced at the center of such cavities, allowing for stronger coupling of trapped ions to the cavity light field by up to a factor of two at optimized parameters compared to standard spherical mirror cavities, while simultaneously also enhancing coupling between the cavity and fiber modes.

\section{ACKNOWLEDGMENTS}

The authors acknowledge financial support from EPSRC through the UK National Quantum Technologies Programme, project EP/M013243/1 "Networked Quantum Information Technologies (NQIT)" and project EP/M013294/1 "UK Quantum Technology Hub for Sensors and Metrology", and through project EP/J003670/1.

The data used in this work is accessible online at the University of Southampton Institutional Research Repository under the Creative Commons Attribution license http://dx.doi.org/10.5258/SOTON/393153

\section{REFERENCES}

[1] Maiwald, R., Leibfried, D., Britton, J., Bergquist, J. C., Leuchs, G. and Wineland, D. J., "Stylus ion trap for enhanced access and sensing," Nat. Phys. 5(8), 551-554 (2009).

[2] Biercuk, M. J., Uys, H., Britton, J. W., VanDevender, A. P. and Bollinger, J. J., "Ultrasensitive detection of force and displacement using trapped ions," Nat. Nanotech. 5(9), 646-650 (2010).

[3] Ludlow, A. D., Boyd, M. M., Ye, J., Peik, E. and Schmidt, P. O., "Optical atomic clocks,” Rev. Mod. Phys. 87(2), 637-701 (2015).

[4] Kimble, H. J., "The quantum internet," Nature 453(7198), 1023-1030 (2008).

[5] Blatt, R. and Roos, C. F., "Quantum simulations with trapped ions," Nat. Phys. 8(4), 277-284 (2012).

[6] Hunger, D., Steinmetz, T., Colombe, Y., Deutsch, C., Hänsch, T. W. and Reichel, J., "A fiber Fabry-Perot cavity with high finesse," New J. Phys. 12, 65038 (2010).

[7] Takahashi, H., Wilson, A., Riley-Watson, A., Oručević, F., Seymour-Smith, N., Keller, M. and Lange, W., “An integrated fiber trap for single-ion photonics," New J. Phys. 15, 53011 (2013).

[8] Podoliak, N., Takahashi, H., Keller, M. and Horak, P., "Comparative numerical studies of ion traps with integrated optical cavities," Phys. Rev. Appl. 6, 044008 (2016).

[9] Podoliak, N., Takahashi, H., Keller, M. and Horak, P., submitted (2016).

[10] Keller, M., Lange, B., Hayasaka, K., Lange, W. and Walther, H., "Continuous generation of single photons with controlled waveform in an ion-trap cavity system," Nature 431, 1075-1078 (2004).

[11] Seidelin, S., et al., "Microfabricated surface-electrode ion trap for scalable quantum information processing," Phys. Rev. Lett. 96(25), 253003 (2006).

[12] Kleckner, D., Irvine, W. T. M., Oemrawsingh, S. S. R. and Bouwmeester, D., "Diffraction-limited high-finesse optical cavities," Phys. Rev. A 81(4), 043814 (2010). 